\title{
On the Design of Beam Absorbers for the SSC
}

\author{
Brett Parker \\ Accelerator Design and Operation Division \\ Superconducting Super Collider Laboratory \\ 2550 Beckleymeade Avenue \\ Dallas, TX 75237, USA
}

\section{Abstract}

The $20 \mathrm{TeV}$ beam absorbers for the Superconducting Super Collider (SSC) present a formidable design challenge. Protons from the SSC will have: 20 times the energy, be 20 times harder to bend, and be distributed with a natural transvers-size $\sqrt{20}$ times smaller than from all previous accelerators. This paper concentrates on the thermo-physical demands made on a beam backstop in terminating $20 \mathrm{TeV}$ protons. In particular radiation-shielding, logic, control, and beam diagnostic requirements will not be discussed[1]. We will report on Monte Carlo simulations, made using the MARS10 code of N. Mokhov[2], which provides a basis for evaluating beam spreading and painting scenarios. The merits of various standard painting schemes are then discussed. Finally we present some new options for spreading the beam spot which are currently under investigation.

\section{INTRODUCTION}

The SSC parameters relevant to the design of beam absorbers are shown in Table 1. Note that the product of the energy per proton and the number of protons per beam gives a circulating energy of 420 megajoules for each collider ring. This kinetic energy is roughly equivalent to the amount dissipated in an 850 car freeway chain-reaction accident; obviously this much energy must be absorbed in a controlled, safe and reliable way.

Table 1.

SSC Parameters Relevant to Beam Absorber Design

\begin{tabular}{ll}
\hline Proton Energy & $2-20 \mathrm{TeV}$ \\
Design Luminosity & $10^{33}$ \\
Circumference & $87.12 \mathrm{~km}$ \\
Revolution Time & $290 \mu \mathrm{s}$ \\
Abort Gap & $3 \mu \mathrm{s}$ \\
Fractional Momentum Spread & $\sim 6 \times 10^{-5}$ \\
Bunch Length & $5-6 \mathrm{~cm}$ \\
Bunch Spacing & $5 \mathrm{~m}$ \\
Number of Bunches Per Ring & 17,424 \\
Protons Per Bunch & $3 / 4 \times 10^{10}$ \\
Protons Per Ring & $1.3 \times 10^{14}$ \\
\hline
\end{tabular}

The beam-abort system is specified to be fast-acting and capable of single-turn $(-300 \mu \mathrm{s})$ extraction of the entire beam from a ring within three turns of the generation of an abort signal[1]. Fast kicker magnets will dispatch the beam through

\footnotetext{
* Operated by Universities Research Association, Inc., for the U.S. Department of Energy under Contract No. DE-AC0289ER40486. U.S. Government work not protected by U.S. Copyright.
}

the field-free region of a string of Lambertson-style septum magnets, down a separate $\sim 2 \mathrm{~km}$ long channel and into a multi-layer beam backstop. The central backstop-core will consist of graphite $10 \mathrm{~m}$ in extent and $2 \mathrm{~m}$ in diameter. Surrounding the graphite will be additional radiation-shielding and monitoring devices. Graphite will be used for the coreregion both to diminish the long-term production of residual radioactivity and to maximize design-robustness (by longitudinally spreading the shower energy-deposition).

\section{METHODOLOGY}

The high-energy cascade showers resulting from $20 \mathrm{TeV}$ protons are simulated using the MARS series of computer code of N. Mokhov[2]. The current version, MARS 12, runs efficiently under VMS ${ }^{\mathrm{TM}}$, DOS and UNIX ${ }^{\mathrm{TM}}$ operating systems on several different computers. The MARS code reproduces existing inclusive particle-spectra from Tevatron energies through thermal-neutron capture[3,4]. A comparison, shown in Figure 1, made between MARS and the codes FLUKA87[5] and CASIM[6] exhibits good agreement[4].

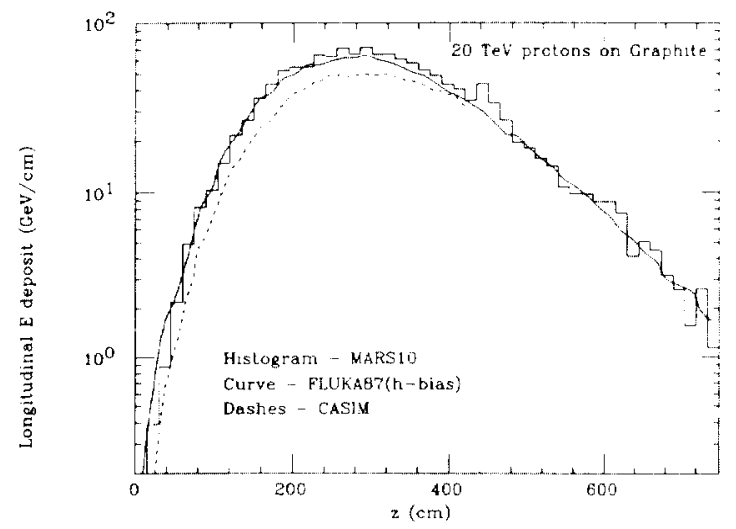

Fig. 1. The longitudinal energy deposition $(\mathrm{GeV} / \mathrm{cm})$ for $20 \mathrm{TeV}$ protons incident on graphite calculated using MARS10 (histogram), FLUKA87 (curve) and CASIM (dash)[4]

Recent calculations, made with a new GHEISHA/GEANT version, are also in agreement with MARS results[7]. Since the physics of shower-cascades scales as $\log (s),(s=$ total energy squared) our predictions for energy-deposition in the beam backstop should be reliable; this is in contrast to the situation for processes, which are much more sensitive to details of exclusive reaction channels, such as muonproduction at the SSC interaction points. 
We present results for the instantaneous temperature rise, derived from a material's thermo-physical properties (i.e. enthalpy reserve $=$ heat needed to raise material's temperature) under the premise that the energy is deposited everywhere at the same instant in time. Such an approximation is suitable for the short, $290 \mu \mathrm{s}$, beam spill time and facilitates the interpretation of beam heating in terms of temperature cracking or melting limits.

\section{RESULTS}

For the core of the beam backstop, it is desirable to choose a material with a high cracking/melting temperature and low density (to spread the shower longitudinally as much as possible); for these reasons, graphite is a natural choice. Carbon's low atomic number, also helps to reduce the amount of long-term induced radioactivity due to spallation fragments. A reference plot of $\Delta T(r, z)$, the radially-symetric temperature distribution, due to a round-Gaussian $(\sigma=10 \mathrm{~cm})$ beam profile incident along the axis of a graphite core, is shown in Figure 2. for $1.3 \times 10^{14}$ protons $\left(=10^{33}\right.$ luminosity).

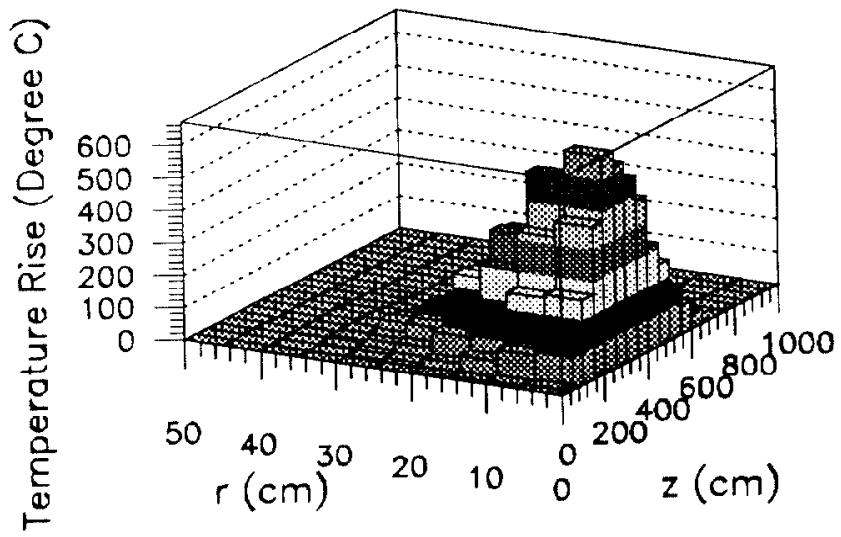

Fig. 2. Radially-symetric temperature rise calculated for $1.3 \times 10^{14}$ protons incident on graphite for a round-Gaussian beam profile and $20 \mathrm{TeV}$. The heating-maximum, $670^{\circ} \mathrm{C}$, occurs on axis at $250-300 \mathrm{~cm}$ longitudinal distance.

Along the $z$-axis, where the beam is most intense, the temperature profile follows the longitudinal energy deposition curve shown in Figure 1 and away from the axis the temperature rise is modulated by the assumed Gaussian beam profile. For this and subsequent comparisons, in each case, we have made simulations for $17 \mathrm{k}$ incident protons which yields few percent statistical errors at the shower maximum. Note that as the hadronic (and electromagnetic) shower starts, there is a rapid rise in the rate of energy deposition followed by a maximum that is reached about $3 \mathrm{~m}$ (or 7 interaction lengths) spanning a length of $3 \mathrm{~m}$.

For a fixed beam intensity, the variation of the maximum instantanenus-heating with heam size is shown in Figure 3. Since a material's specific heat varies with temperature, the integration of its specific heat curve, its enthalpy reserve, will not be a linear function of temperature change for large changes. The convolution, for graphite, between its enthalpy reserve and a $\sigma^{2}$ variation of beam size gives an approximate $\sigma^{-4 / 3}$ scaling. A relevant design limit $\left(\Delta T=2300^{\circ}\right.$ for graphite) known as the fracture limit is indicated on Figure 3.

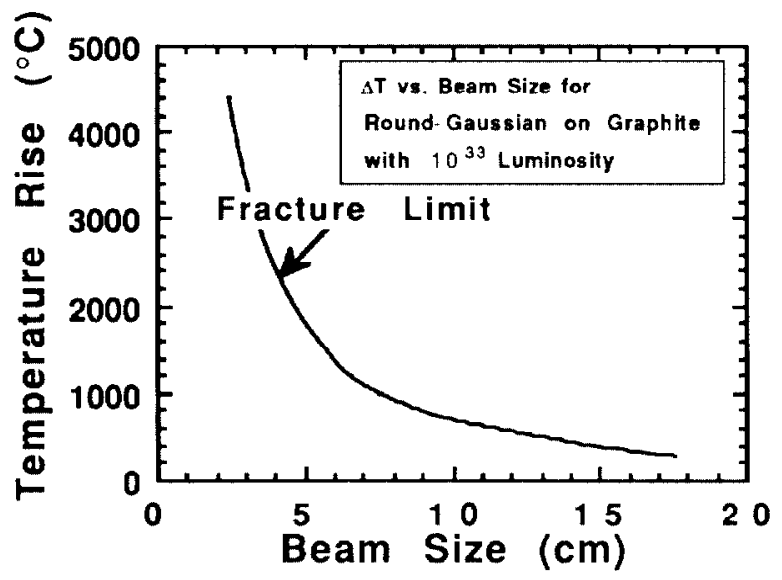

Fig. 3. Temperature rise as a function of beam $\sigma$ for $1.3 \times 10^{14}$ protons incident on graphite at $20 \mathrm{TeV}$.

This limit, which is lower that graphite's melting point, comes from thermal shock considerations[8]. It is prudent to maintain an operating margin that reflects systematic uncertainties in the simulation and so as not to preclude future luminosity (via beam current) upgrades.

A $10 \mathrm{~cm}$ beam spot, obtained from magnetic quadrupolefocusing, satisfies the above; however, a prohibitive amount of defocusing strength is required because of the SSC ( . .1 $\mathrm{mm}$ ) beam size and momentum. An extension is to defocus asymmetrically in one dimension (horizontal) and sweep the beam spot with a slow kicker magnet in the other transvers dimension (vertical). Unfortunately, the presence of too large a magnification in any plane drives a need for unacceptably large abort line apertures. One is soon led to consider beam painting schemes. In Figure 4 we show a beam profile that corresponds to the baseline CDR[1] "spiral kicker" scheme. For the CDR approach one uses two set of orthoginal, fast damped-magnetic kickers, along with limited magnification, to move the beam spot on the backstop face. Note that the damping causes beam pileup on the inner face of the spiral which sets an inner limit for painting. Also if the phase relationship of between the orthoginal kickers of $90^{\circ}$ is not maintained, an oval beam spot (as shown in Figure 5) results.

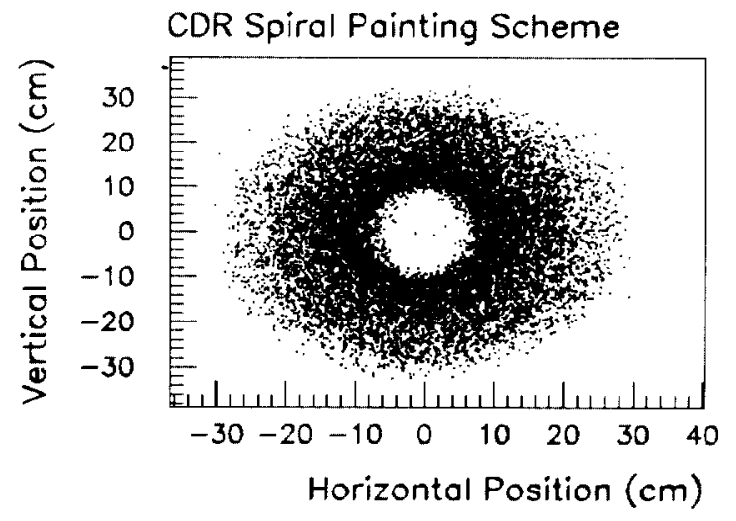

Fig. 4. Nominal beam profile for CDR spiral painting scheme. 


\section{Future prospects \& Conclusions}

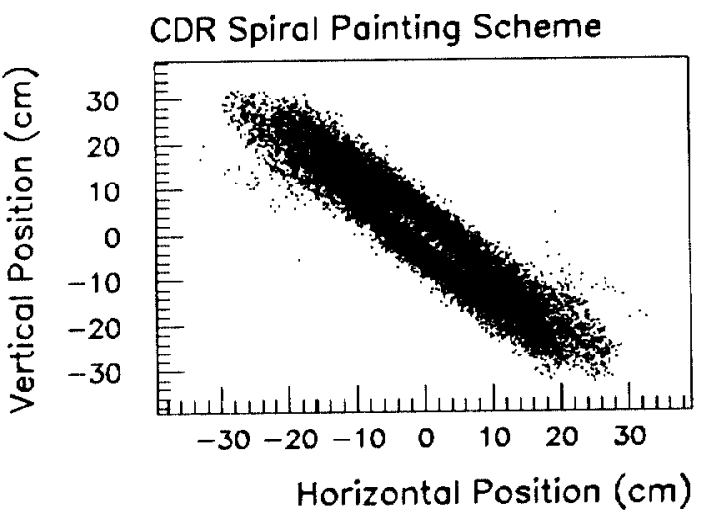

Fig. 5. CDR spiral painting with phase slippage.

A raster-pattern (shown in Figure 6) can be created via a combination of fast and slow kickers. Such a painting scheme with less needed fast-kicker strength and vastly reduced sensitivity to phase errors, is expected to be more reliable than the CDR spiral plan; however, there is some beam pile up near the outer edges of the raster pattern.

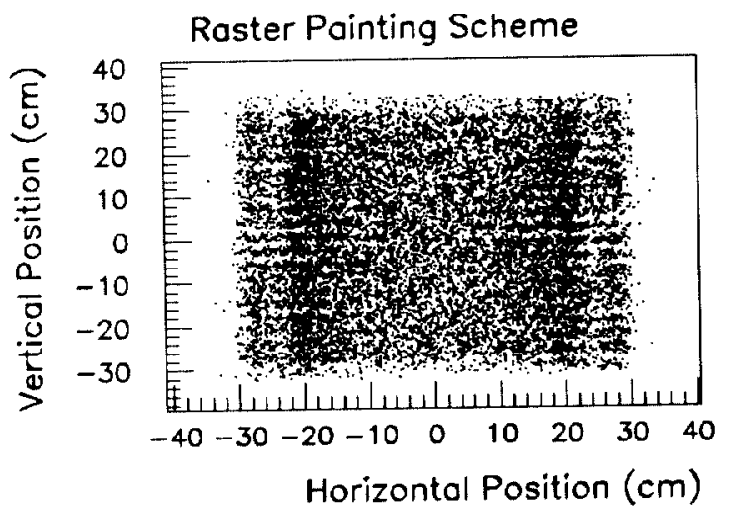

Fig. 6. Beam profile for raster painting scheme.

Attempts have been made to mitigate this edge concentration by the addition of nonlinear (sextupole, octupole etc.) magnetic elements to the abort channel in a manner similar to that suggested by B. Blind[9]. The addition of nonlinearities, while helpful in spreading (or folding) the pattern edges, reintroduces some sensitivity to initial beam position offsets at the beginning of the abort channel. The addition of a short sextupole would be beneficial if it is found convenient to have moderate kicker damping ( $20 \%$ over the $300 \mu$ s spill). A properly placed sextupole could be used to "square up" the resulting trapazoidal-shape raster pattern (Figure not shown).

It has been suggested to place a series of thin targets well upstream of the beam backstop to further spread the beam; however, simulations show that a fcw radiation lengths of material will add little, about a $\mu$ radian, to the beam divergence at $20 \mathrm{TeV}$. Such a plan would place an intense radiation source undesirably close to the collider tunnel.
At present a raster-scan based technique is the most promising and budget-friendly approach. Future detailed design work calculations will revisit: shielding and radiation requirements, muon vectors etc. Also work is underway to investigate the feasibility of using unconventional components to eliminate either the kicker magnets or the conventional quadrupoles.

Preliminary studies using the code TBCI[10] suggest it might be possible to use the beam's wake-field to excite transversedeflection modes of a passive beam-tube insert. We are examining candidates in terms of enhancing wake-field effects. If a suitable, simple and inexpensive structure is found, one could imagine repeating said structure many $\left(10^{3}-10^{4}\right)$ times near the beginning of the abort channel. With sufficient repetition and enough bunches participating (a modest faction of 17,000), a useful increase of beam emittance may be possible. Such a passive "beam-spoiler" has the advantage of being fail safe. Also the magnitude of the effect would automatically track increases in beam current for upgrades.

For the conventional quadrupole magnets, which must be powered continuously, one might consider using a plasma lens[11] or pulsed quadrupole[12]. Some of the very effects that limit the applicability of these devices (beam-plasma scattering and nonlinear aberrations) would be beneficial in this case. The tradeoff for such devices would be between reduced power consumption vs. an increased system complexity. Maybe the ultimate (pie in the sky) beam-spoiler could use a self-excited plasma inside a self-excited cavity; who knows.

\section{References}

[3] N. Mokhov, Sov. J. Part. Nucl., Vol. 18(5), pp. 408-426, 1987.

[4] N. Mokhov, in "Radiation Levels in the SSC Interaction Regions," SSC-SR-1033, pp 303-311, 1988.

[5] P. Aarnio et al., CERN TIS-RP/190, 1987.

[6] A. Van Ginneken, Fermilab FN-272, 1975.

[7] N. Mokhov and Igor Azhgirey, Institute for High Energy Physics, Protvino, USSR, Private Comm.

[8] J. Kidd et.al., IEEE Transactions on Nuclear Science, Vol. NS-28, No. 3, June 1981.

[9] B. Blind, "Generation of a Rectangular Beam Distribution for Irradiation of the Accelerator Production of Tritium Target," Los Alamos preprint LA-UR-90-2759, 1990.

[10] Th. Weiland, computer code TBCI, DESY, 2000 Hamburg 52, Notkestr. 85, Germany, copyright 1986.

[11] G. Le Dallic et al., "First Test Results from the New CERN Plasma Lens," preprint; J. Cristiansen et al., CERN Report, CERN/PS/84-10, 1984.

[12] M. Modena, P. Sievers, CERN/AT-MA/90-22, 1990. 